\title{
Type and severity of septal deviation are not related with the degree of subjective nasal obstruction*
}

\author{
Sarah Verhoeven, Bert Schmelzer \\ Department of Otolaryngology-Head and Neck Surgery, ZNA Middelheim Hospital, Antwerp, Belgium
}

Rhinology 54: 355-360, 2016

DOI:10.4193/Rhino15.226

*Received for publication:

August 18, 2015

Accepted: March 31, 2016

\begin{abstract}
Background: Septoplasty is a frequently performed operation by otolaryngologists to relieve nasal obstruction complaints. When objective measurements tools are not available, preoperative decision-making is based on careful clinical examination. Our aim was to evaluate the relationship between type and severity of septal deviation and patient-reported nasal obstruction.
\end{abstract}

Methodology: 196 Patients of a general otolaryngology population were included. Patients indicated subjective nasal obstruction experienced during the past 1 month on a Visual Analog Scale (VAS). Patients underwent clinical examination to evaluate type and severity of septal deviation. We compared clinical examination findings with patient's VAS.

Results: We did not find a statistical difference of VAS score between the different types and severities of septal deviation. In $33.9 \%$ of the cases without septal deviation, there was a corresponding VAS score of 0. In $26,8 \%$ of the cases with septal deviation, there was no complaint of nasal obstruction (VAS $=0$ ). In $7,4 \%$ of the cases, there was moderate to severe nasal obstruction complaint (VAS > 4) though no septal deviation was found.

Conclusions: These results demonstrate that patient-reported nasal obstruction varies greatly among patients with similar type of deviation and similar degree of deviation. Classification of septal deviation into type and severity cannot predict the degree of subjective nasal obstruction. Therefore, the decision to proceed to septoplasty has to be thoughtful, with as much as information, based on the combination of patient's history, clinical examination, surgeon's experience and cautious interpretation of objective measurement tools.

Key words: septoplasty, clinical examination, nasal obstruction, visual analog scale

\section{Introduction}

Nasal airway obstruction is a common health complaint that affects all age groups and reduces quality of life (1). The etiology of nasal airway obstruction is determined by different conditions of which a deviated nasal septum is often diagnosed by otolaryngologists during clinical examination. Currently no gold standard exists for objective assessment of nasal patency and more specifically to diagnose nasal septal deviation. Different measurement tools for the diagnosis of nasal septal deviation were reviewed by Aziz et al. in $2014^{(2)}$. They concluded that acoustic rhinometry, rhinomanometry and nasal spectral sound analysis add little value to identify presence, location and severity of nasal septal deviation. Furthermore the correlation between currently existing objective tests for nasal airway patency and the patient's symptoms remains controversial ${ }^{(3)}$. Many sinonasal surgical procedures are carried out annually of which septoplasty is one of the most frequently performed operations by otolaryngologists worldwide. According to the Belgian Institute for Public Sickness Insurance and Handicaps (RIZIV) 13832 septoplasty procedures were conducted in 2013 (approximately 1.3 cases per 1,000 persons). In the USA, septoplasty is the third most common surgical procedure carried out by otolaryngologists ${ }^{(4)}$. In 2006 , about 260,000 septoplasty procedures were performed in ambulatory setting in the USA 
(approximately 0.8 cases per 1,000 persons) ${ }^{(5)}$.

The decision to proceed to septoplasty is often based on history taking and the surgeon's clinical examination findings and experience alone, firstly because of the poor correlation between patient's symptoms and objective measurement tools, and secondly because objective measurement tools are not always present in every otolaryngology practice. Clinical assessment still proves to be an accurate predictor of patients who are likely to fail medical treatment and will need septoplasty ${ }^{(6)}$. Despite that clinical examination remains the cornerstone to decide for surgical intervention, only a few studies investigate the relationship between clinical findings and subjective nasal obstruction. To our knowledge, no previous study investigated the relationship between the degree of subjective nasal obstruction complaint and the specific type of septal deviation. Therefore the aim of our study was to evaluate the relationship between type of septal deviation evaluated during clinical examination and the degree of subjective nasal obstruction, in a general otolaryngology patient population. A universally accepted classification system for nasal septal deviation is still lacking. In our study we evaluated the type of septal deviation according to Mladina et al. ${ }^{(7)}$. Furthermore we evaluated the relationship between the degree of subjective nasal obstruction and the severity of septal deviation, according to Salihoglu et al. ${ }^{\left({ }^{(8)} \text {. }\right.}$

\section{Materials and methods}

The study protocol was approved by the ethics committee of ZNA Middelheim hospital. Patients, aged 18 years or older, presenting for various ear, nose and throat complaints at our department, were asked to fill out a questionnaire and undergo clinical examination as part of a routine otolaryngology examination. From August to December 2014, 285 patients participated.

\section{Questionnaire}

The questionnaire asked about conditions which can alter nasal patency such as previous nasal trauma or nasal surgery less than 1 year ago, (self-reported) allergy (to airborne allergens), acute or chronic sinusitis and current use of topical vasoconstrictors. If one of these factors was present, the patient was excluded. Patients indicated subjective nasal obstruction experienced during the past 1 month on a Visual Analog Scale (VAS), right and left nostril separately $(0=$ no nasal obstruction; $10=$ no nasal breathing possible).

\section{Clinical examination}

A general otolaryngology clinical examination was performed, including anterior rhinoscopy to evaluate the nasal cavity and septum, without prior knowledge of the questionnaire results. Only two specialists observed the nasal septum to minimize interobserver variability. Nasal endoscopy with or without topi-

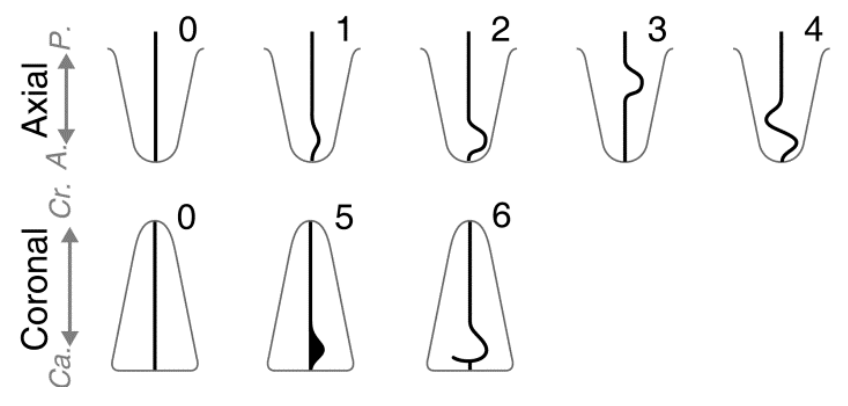

Figure 1. Overview of different types of septal deviation. Type 0 represents a straight septum. Type 1 to 4 are vertical deviations, presented in axial plane. Type 5 and 6 are horizontal deviations, presented in coronal plane. A. Anterior, P. Posterior, Ca. Caudal, Cr. Cranial

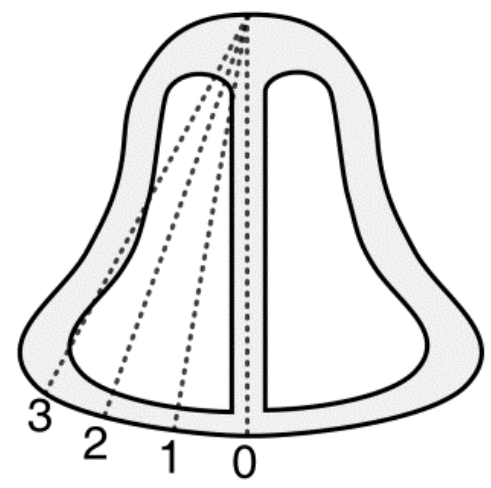

Figure 2. Overview of different degrees of septal deviation. Score 0 corresponds with no significant deviation, score 1 with 1/3rd obstruction, score 2 with 2/3rds obstruction and score 3 with complete obstruction.

cal decongestants was performed if septal deviation type and severity were not evident after anterior rhinoscopy alone, for example in cases of posterior deviations.

Type of septal deviation was classified according to Mladina et al. ${ }^{(7)}$ (Figure 1). Mladina's classification considers the functional aspect of the nasal valve and nasal cavity and describes seven types of septal deviation defined by the sagittal plane. Types 1 to 4 are presented in axial view in figure 1 and are vertical deviations. Type 1 is a vertical deviation anteriorly which does not alter nasal valve region, whereas type 2 does obstruct normal function of the nasal valve. Type 3 is situated at the head of the middle turbinate. Type 4 is a combination of type 2 and 3 , each at one side. Type 5 and 6 are presented in coronal view in figure 1 and categorized as horizontal deviations. Type 5 corresponds with a horizontal spur on one side. Type 6 is comparable to type 5 with on the opposite side a unilateral intermaxillary bone wing with a 'gutter' between it. Type 7 is a combination of two or more types, though we did not apply this type in our study. For each nostril, we took the most significant deviation into account; 
otherwise many deviations would end up being classified as a combination of several deviations types, namely Mladina type 7 . We added type 0 to Mladina's classification, which represents no septal deviation.

Since Mladina's classification does not represent the severity of septal deviation, we added a scale of severity of septal deviation, according to Salihoglu et al. ${ }^{\left({ }^{8}\right)}$ (Figure 2). We gave a score from 0 to 3 for right and left nostril separately. Score 0 corresponds with no significant deviation. So it might be that a minor deviation, for example a small vertical ridge, is classified as type 1 according to Mladina but with a severity score of 0 according to Salihoglu. If $1 / 3$ rd of the nasal cavity was obstructed, we gave a score 1 ("mild deviation"). If $2 / 3$ rds of the nasal cavity was obstructed we gave a score 2 ("moderate deviation"). If the nasal cavity was totally obstructed, we gave a score 3 ("severe deviation").

\section{Statistics}

Data analysis was performed with IBM SPSS Statistics 22 for Windows. All tests were conducted at the $5 \%$ significance level. Continuous variables were checked for normality by Kolmogorov-Smirnov test. Association between the outcomes of right and left nostril were tested by Wilcoxon signed ranks test for severity of septal deviation and VAS, and by Pearson's Chi-square test concerning type of septal deviation. Comparison of VAS score between the group without and with septal deviation was performed with independent-samples T-test. We compared VAS score for the different categories of septal deviation severity and type (Kruskal-Wallis $\mathrm{H}$ test, post-hoc Mann-Whitney $\mathrm{U}$ test with Bonferroni correction).

\section{Results}

\section{Exclusion}

284 Patients filled out the questionnaire and underwent clinical examination. 88 Patients were excluded because of potential confounders: (self-reported) allergy $(N=29)$, sinonasal surgery or nasal trauma less than 1 year ago $(\mathrm{N}=10)$, current use of topical decongestants $(\mathrm{N}=10)$, inferior turbinate hypertrophy $(\mathrm{N}=10)$, septal perforation ( $N=9$ ), incomplete questionnaire ( $N$ $=7)$, sinusitis with or without nasal polyps $(N=7)$, nasal valve dysfunction ( $N=3)$, concha media bullosa $(N=1)$, concha media amputation $(\mathrm{N}=1)$, adenoid cystic carcinoma $(\mathrm{N}=1)$. Thus 196 patients were included and their data were statistically analysed.

\section{Patient population}

Mean age was 60,26 years (SD $=17,719$; range $20-97$ ) and male/ female ratio was $85 / 111$. Right and left nostrils were separately and independently evaluated. No significant association was found for the outcome of right and left nostril for type of septal deviation $(p=0,163)$, severity of septal deviation $(p=0,054)$ and

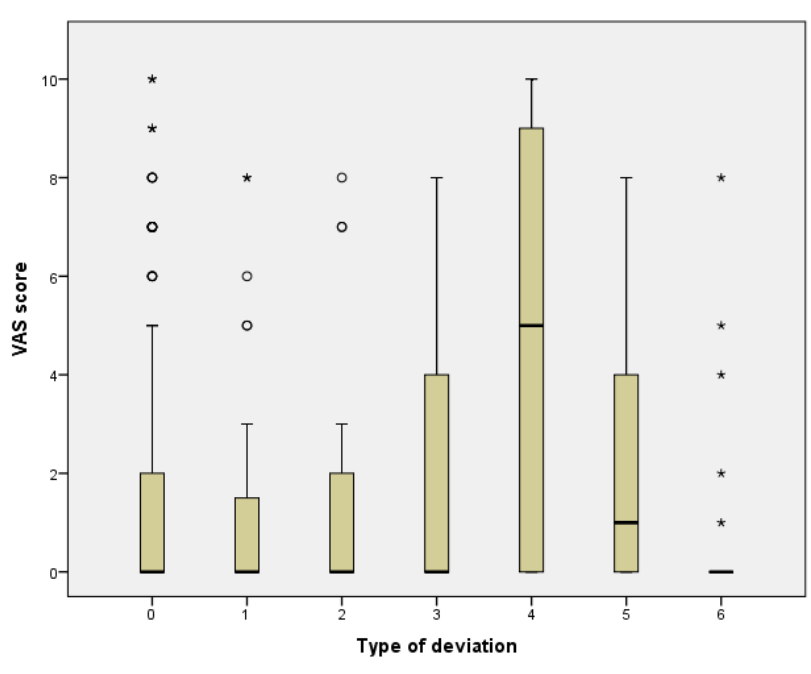

Figure 3. Boxplot showing the VAS score among the different Mladina categories. Median, 1st quartile, 3rd quartile, minimum and maximum values excluding outliers are displayed in the boxplot. Circles and asterisks represent mild and extreme outliers respectively. All types of deviation have a large distribution of VAS score and type 4 corresponds with the highest median VAS score.

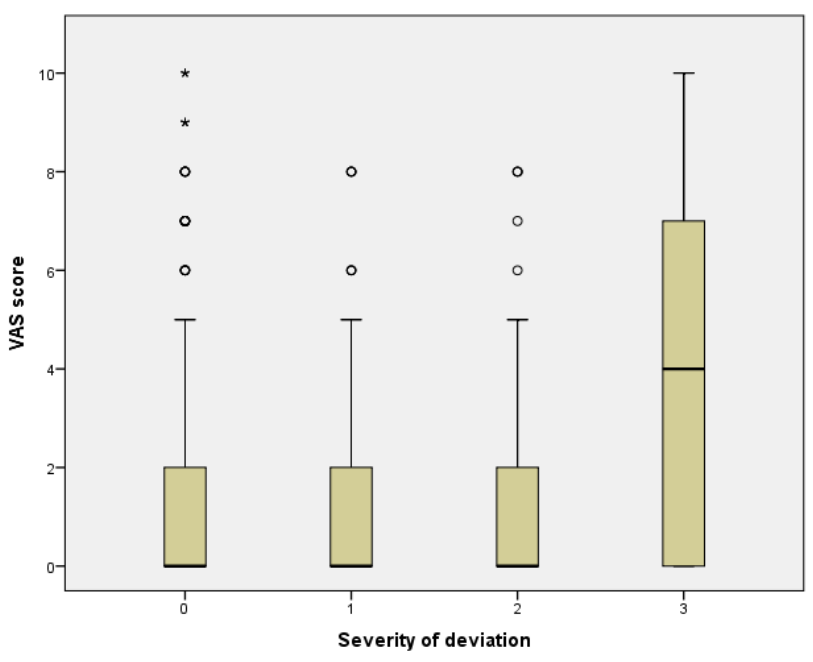

Figure 4. Boxplot showing the VAS score among the different Salihoglu categories. Median, 1st quartile, 3rd quartile, minimum and maximum values excluding outliers are displayed in the boxplot. Circles and asterisks represent mild and extreme outliers respectively. All types of severity have a large distribution of VAS score and score 3 corresponds with the highest median VAS score.

VAS score $(p=0,615)$. Therefore further analysis was done for unilateral data $(\mathrm{N}=392)$.

\section{Type of septal deviation versus VAS}

The mean VAS score in the total study population was 1,61 (SD =2,576; range 0-10). The mean VAS score in the group without septal deviation (type 0 ) was 1,41 (SD = 2,468; range $0-10$ ) and for the group with septal deviation (type 1 until 6) 1,82 (SD = 
Table 1. Mladina categories with corresponding percentages, mean VAS score with standard deviation (SD) and range (minimum-maximum).

\begin{tabular}{ccc|}
$\begin{array}{c}\text { Mladina type of } \\
\text { septal deviation }\end{array}$ & $\%$ & $\begin{array}{c}\text { Mean VAS (SD; } \\
\text { minimum-maximum) }\end{array}$ \\
\hline 0 & 50,8 & $1,41(2,468 ; 0-10)$ \\
1 & 7,1 & $1,39(2,544 ; 0-8)$ \\
2 & 4,3 & $1,71(2,823 ; 0-8)$ \\
3 & 9,2 & $1,58(2,523 ; 0-8)$ \\
4 & 2,3 & $4,67(4,416 ; 0-10)$ \\
5 & 20,4 & $2,05(2,5 ; 0-8)$ \\
6 & 5,9 & $0,87(2,052 ; 0-8)$ \\
\hline
\end{tabular}

Table 2. Salihoglu categories with corresponding percentages, mean VAS score with standard deviation (SD) and range (minimum-maximum).

\begin{tabular}{|lcc|}
\hline $\begin{array}{c}\text { Severity of septal } \\
\text { deviation }\end{array}$ & $\%$ & $\begin{array}{c}\text { Mean VAS (SD; } \\
\text { minimum-maximum) }\end{array}$ \\
\hline 0"no" & 56,4 & $1,51(2,489 ; 0-10)$ \\
\hline 1"mild" & 22,2 & $1,39(2,325 ; 0-8)$ \\
\hline 2"moderate" & 15,1 & $1,46(2,402 ; 0-8)$ \\
\hline 3"severe" & 6,3 & $3,6(3,686 ; 0-10)$ \\
\hline
\end{tabular}

Table 3. Crosstab with percentages of VAS categories versus septal deviation severity.

\begin{tabular}{lcccc}
$\begin{array}{c}\text { Septal devia- } \\
\text { tion severity }\end{array}$ & $\begin{array}{c}\mathbf{0} \\
\text { "no" }\end{array}$ & $\begin{array}{c}\mathbf{1} \\
\text { "mild" }\end{array}$ & $\begin{array}{c}\mathbf{2} \\
\text { "moder- } \\
\text { ate" }\end{array}$ & $\begin{array}{c}\mathbf{3} \\
\text { "severe" }\end{array}$ \\
\hline VAS "no" & 33,9 & 14,3 & 9,7 & 2,8 \\
\hline 1-4 "mild" & 15,1 & 4,6 & 3,1 & 0,5 \\
\hline 5-7 "moderate" & 4,6 & 2,3 & 1,5 & 2,0 \\
\hline 8-10"severe" & 2,8 & 1,0 & 0,8 & 1,0 \\
\hline
\end{tabular}

Table 4. Simplified crosstab with percentages of VAS categories (0-4; 5-10) versus septal deviation (no septal deviation; present septal deviation mild to severe).

\begin{tabular}{|c|c|c|}
\hline $\begin{array}{r}\text { Septal deviation } \\
\text { severity }\end{array}$ & $\begin{array}{c}0 \\
\text { "no" }\end{array}$ & $\begin{array}{c}1 \text { to } 3 \\
\text { "mild to severe" }\end{array}$ \\
\hline 0 to 4 "no to mild" & 49,0 & 35,0 \\
\hline $\begin{array}{l}5 \text { to } 10 \\
\text { "moderate to severe" }\end{array}$ & 7,4 & 8,6 \\
\hline
\end{tabular}

2,674; range 0-10). There was no statistical significant difference in VAS score between the group with and without septal deviation $(p=0,114)$. Percentages of different deviation types with corresponding mean VAS score are shown in Table 1. A boxplot of the VAS score among the different Mladina categories is shown in Figure 3. Kruskal-Wallis H-test showed that the distribution of VAS score is not the same across the categories of septal deviation type $(p=0,016)$, although post-hoc MannWhitney $\mathrm{U}$ test with Bonferroni correction demonstrated no significant difference between two categories.

\section{Severity of septal deviation versus VAS}

In 56,4\% no significant septal deviation was found. The mean VAS score in the group without significant septal deviation (severity score 0 ) was 1,51 (SD $=2,489$; range $0-10$ ) and for the group with septal deviation (severity score 1 until 3) 1,74 (SD = 2,687; range 0-10). There was no statistical significant difference in VAS score between the group with and without significant septal deviation $(p=0,391)$. Percentages of severity scores of septal deviations with corresponding mean VAS score are shown in Table 2. A boxplot of the VAS score among the different Salihoglu categories is shown in Figure 4. Kruskal-Wallis H-test showed that the distribution of VAS score is not the same across the categories of septal deviation severity $(p=0,044)$. Post-hoc Mann-Whitney $\mathrm{U}$ test with Bonferroni correction demonstrated only a significant difference between the group mild and severe septal deviation $(p=0,007)$.
Next, the VAS scores were clustered into 4 categories; no (0), mild (1-4), moderate (5-7) and severe (8-10) nasal obstruction complaint. A crosstab with percentages of VAS categories versus septal deviation severity is shown in Table 3. In 33.9\% of the cases without septal deviation, there was a corresponding VAS score of 0 . In $26,8 \%$ of the cases with septal deviation, there was no complaint of nasal obstruction (VAS $=0$ ). Furthermore 3,3\% of the severe septal deviation group (score 3 ) had no or mild nasal obstruction complaint (VAS score $<5$ )

Finally, the VAS and severity scores were each further clustered into 2 categories of which the corresponding percentages are represented in Table 4. In 8,6\% with a present septal deviation (score 1,2 or 3 ), there was moderate to severe complaint (VAS > 4). Lastly, 7,4\% had moderate to severe nasal obstruction complaints (VAS > 4) though no septal deviation was found.

\section{Discussion}

Septoplasty is a frequently performed operation by otolaryngology surgeons worldwide to relieve nasal airway obstruction complaints. The aim of this study was to evaluate the relationship between type and severity of septal deviation evaluated during clinical examination, and degree of subjective nasal obstruction in a general otolaryngology patient population.

The mean VAS score for nasal obstruction in our total study population is $1,61(S D=2,576$; range $0-10)$. This low average score 
corresponds with a very mild nasal obstruction complaint which is probably in most cases not enough interfering with daily activity to search for medical advice. In 2014, a systematic review of Rhee et al. found a weighted average VAS score (weighted for sample size) for the general population of $4,6(S D=2,6)$ which is remarkably higher than in our study population ${ }^{(9)}$. Rhee's review included three studies (total population $\mathrm{N}=3063$ ) with a middle aged population on average; Jones et al. reported a mean age of 32 (range 20-48), Lam et al. reported a median age of 46 (range 16-87) and Kjaergaard et al. reported a median age of 46 (range $16-87)^{(10-12)}$. In our study there is a considerably higher mean age of 60,26 (SD = 17,719; range 20-97). The higher mean age in our study population might explain the lower mean VAS score. Aging can cause alterations in nasal physiology and appearance (size and shape). In 2010, a study showed that nasal patency improves across the lifespan as seen in acoustic rhinometry ${ }^{(13)}$. This enlargement of nasal cross-sectional area was attributed to mucosal atrophy. Secondly, this study showed that nose-related quality of life is not affected by older age according to outcome instruments, such as the Nose Obstruction Symptom Evaluation (NOSE) questionnaire. In an epidemiologic retrospective study of more than 11000 patient charts, there was no significant age-related increase in nasal obstruction complaint ${ }^{(14)}$. Another study showed that younger people reported a higher degree of nasal obstruction ${ }^{(15)}$. Age-related changes usually develop slowly, therefore aging people become gradually habituated to it and do not often report nasal changes such as nasal obstruction.

In our study, each septal deviation type according to Mladina results in a large distribution of VAS scores and there is no statistical difference of VAS score between the different types. Type 4 corresponds with the highest mean VAS score of 4,76, which is not surprisingly because of the underlying anatomy of type 4, which is in fact a S-shaped deviation with deformity at both septal sides. We have to be aware of the small simple size of type 4 (2,3\%). According to Salighoglu's severity classification, we found in $56,4 \%$ no significant septal deviation, whereas according to Mladina's type classification, septal deviation was absent in 50,8\%. This difference in percentage can be explained because some minor deviations, for example a small horizontal septal spur (Mladina type 5), did not obstruct the nasal cavity for at least $1 / 3$ rd and thus was classified as severity 0 according to Salihoglu. The different severity groups correspond with a great range of VAS scores. The most severe deviation group (score 3 ) corresponds with the highest mean VAS score of 3,6 (SD =6,686; $0-10)$, which is actually not notably high when the nasal cavity is totally obstructed. In general, these results demonstrate that the subjective patient-reported complaint varies greatly among patients with similar type of deviation and similar degree of deviation. Furthermore, type and severity of septal deviation are not related with the degree of subjective nasal obstruction.

These results are not surprising because firstly, clinical examination is subjective and vulnerable to examination bias, and secondly, nasal obstruction complaint is subjective and variable as well. Given the subjective nature of this assessment, the poor correlation between existing objective tests and patient's symptoms, and the complex nasal geometry, it is not surprising that reported surgical success rates of septoplasty are ranging from $23 \%$ to $84 \%{ }^{(16,17)}$. Moreover, the probability of substantial symptom relief gradually decreases with time ${ }^{(18)}$. Already in 1989 , Jessen et al. published that close to half of their patients reported nasal airway obstruction symptoms 9 months after septoplasty, and only about one-fourth were symptom-free after 9 years ${ }^{(19)}$. The most recent publication concerning long-term symptom relief demonstrated that $53 \%$ of the patients have remained or worsened symptoms at 34-70 months after septoplasty ${ }^{(17)}$. Even though septoplasty is one of the most frequently performed operations by otolaryngology surgeons worldwide, the overall results for long-term relief of nasal obstruction and associated improvement of quality of life are unsatisfactory.

A VAS score of 0 was found in $33,9 \%$ of the cases without septal deviation. In $26,8 \%$ of the cases with significant septal deviation (score $1,2,3$ ) there was no complaint of nasal obstruction (VAS $=0$ ). Salihoglu et al. reported a similar percentage of $29,73 \%{ }^{(8)}$. This group represents a substantial number of patients who will not search for medical advice despite their septal deviation. On the other hand, 8,6\% with significant septal deviation (score 1,2, 3) had moderate to severe complaints (VAS score $>4$ ) and these patients might be helped by undergoing septoplasty. Lastly, $7,4 \%$ had moderate to severe nasal obstruction complaints (VAS $>4$ ) though no septal deviation was found. The latter group is likely to seek for medical advice but nasal obstruction cannot be relieved by septoplasty. A Korean study of 970 patients reported a similar percentage of $6,8 \%$ of nasal obstruction complaint without septal deviation ${ }^{(20)}$.

Clearly, it remains a challenge to distinguish physiological septal deviation from pathological deviation which would benefit from septoplasty. A substantial percentage $(7,4 \%)$ of our study population has moderate to severe complaints though with a normal clinical examination. Here clinical examination fails to pinpoint the cause of nasal obstruction complaint. A reason for the discrepancy between clinical examination and subjective patient assessment might be that perception of nasal patency is more than anatomical deformities alone. In fact, perception of nasal patency is the result of multiple complex physical processes such as heating, humidification, resistance and filtration. Current objective tools such as acoustic rhinometry and rhinomanometry cannot measure all these physical processes. A growing 
number of articles report a poor correlation between nasal obstruction complaint and current objective measurements ${ }^{(3,21)}$. The last two decades, researchers are trying to find new innovative objective measurement tools and there is growing body of evidence that computational fluid dynamics could fill this gap by providing consistent objective measurements of nasal airflow and function ${ }^{(16,22-24)}$. Our findings emphasize the need for new reliable objective measurement techniques, not only for presurgical decision-making but also for evaluation of medical and surgical treatments, research documentation and medico-legal purposes.

\section{Conclusion}

In conclusion, these results demonstrate that patient-reported nasal obstruction varies greatly among patients with similar type of deviation and similar degree of deviation. Classification of septal deviation into type and severity cannot predict the degree of subjective nasal obstruction. Therefore, the decision to proceed to septoplasty has to be thoughtful, with as much as information, based on the combination of patient's history, careful clinical examination, surgeon's experience and cautious interpretation of objective measurement tools.

\section{Authorship contribution}

SV: literature review, data collection, analysis of the results, writing manuscript; BS: idea for research, data collection, critical review.

\section{Conflict of interest}

None.

\section{References}

1. Rhee JS, Book DT, Burzynski M, Smith TL. Quality of life assessment in nasal airway obstruction. Laryngoscope 2003; 113: 11181122.

2. Aziz T, Biron VL, Ansari K, Flores-Mir C. Measurement tools for the diagnosis of nasal septal deviation: a systematic review. J Otolaryngol Head Neck Surg 2014; 43: 11.

3. André RF, Vuyk HD, Ahmed A, Graamans K, Nolst Trenité GJ. Correlation between subjective and objective evaluation of nasal airway. A systematic review of the highest level of evidence. Clin Otolaryngol 2009; 34: 518-525.

4. Siegel NS, Gliklich RE, Taghizadeh F, Chang Y. Outcomes of septoplasty. Otolaryngol Head Neck Surg 2000; 122: 228-232.

5. Bhattacharyya N. Ambulatory sinus and nasal surgery in the United States: demographics and perioperative outcomes. Laryngoscope 2010; 120: 635-638.

6. Sedaghat AR, Busaba NY, Cunningham MJ Kieff DA. Clinical assessment is an accurate predictor of which patients will need septoplasty. Laryngoscope 2013; 123: 48-52.

7. Mladina R. The role of maxillary morphology in the development of pathological septal deformities. Rhinology 1987; 25: 199-205.

8. Salihoglu M, Cekin E, Altundag A, Cesmeci E. Examination versus subjective nasal obstruction in the evaluation of the nasal septal deviation. Rhinology 2014; 52: 122126.

9. Rhee JS, Sullivan CD, Frank DO, Kimbell JS, Garcia GJ. A systematic review of patientreported nasal obstruction scores: defining normative and symptomatic ranges in surgical patients. JAMA Facial Plast Surg 2014; 16: 219-225.

10. Jones AS, Willatt DJ, Durham LM. Nasal air- flow: resistance and sensation. J Laryngol Otol 1989; 103: 909-911.

11. Lam DJ, James KT, Weaver EM. Comparison of anatomic, physiological, and subjective measures of the nasal airway. Am J Rhinol 2006; 20: 463-470.

12. Kjaergaard T, Cvancarova M, Steinsvage SK. Does nasal obstruction mean that the nose is obstructed? Laryngoscope 2008; 118: 1476-1481.

13. Lindemann J, Tsakiropoulou E, Konstantinidis I, Lindemann K. Normal aging does not deteriorate nose-related quality of life: assessment with "NOSE" and "SNOT-20" questionnaires. Auris Nasus Larynx 2010; 37: 303-307.

14. Edelstain DR. Aging of the normal nose in adults. Laryngoscope 1996; 106: 1-25.

15. Udaka T, Suzuki H, Kitamura T, et al. Relationship among nasal obstruction, daytime sleepiness, and quality of life. Laryngoscope 2006; 116: 2129-2132.

16. Rhee JS, Pawar SS, Garcia GJ, Kimbell JS. Towards personalized nasal surgery using computations fluid dynamics. Arch Facial Plast Surg 2011; 13: 305-310.

17. Sundh C, Sunnergren O. Long-term symptom relief after septoplasty. Eur Arch Otorhinolaryngol. 2015 Oct;272(10):2871-5.

18. Ho WK, Yuen AP, Tank KC, Wei WI, Lam PK. Time course in the relief of nasal blockage after septal and turbinate surgery: a prospective study. Arch Otolaryngol Head Neck Surg 2004; 130: 324-328.

19. Jessen M, Iversson A, Malm L. Nasal airway resistance and symptoms after functional septoplasty: comparison of findings at 9 months and 9 years. Clin Otolaryngol Allied Sci 1989; 14: 231-234.

20. Wee JH, Kim DW, Lee JE, et al. Classification and prevalence of nasal septal deformity in Koreans according to two classification systems. Acta Otolaryngol 2012; 132 Suppl 1: $S 52-57$

21. Pawar SS, Garcia GJ, Kimbell JS, Rhee JS. Objective measures in aesthetic and functional nasal surgery: perspectives on nasal form and function. Facial Plast Surg 2010; 26: 320-327.

22. Wen J, Inthavong K, Tu J, Wang S. Numerical simulations for detailed airflow dynamics in a human nasal cavity. Respir Physiol Neurobiol 2008; 161: 125-135.

23. Di MY, Jiang Z, Gao ZQ, Li Z, An YR, Ly W. Numerical simulation of airflow fields in two typical nasal structures of empty nose syndrome: a computational fluid dynamics study. Plos One 2013; 8: 842-843.

24. Ozlugedik S, Nakiboglu G, Sert C, et al. Numerical study of the aerodynamic effects of septoplasty and partial lateral turbinectomy. Laryngoscope 2008; 118: 330-334.

Sarah Verhoeven, MD

Department of Otolaryngology

Head and Neck Surgery

ZNA Middelheim Hospital

Lindendreef 1

2020 Antwerp

Belgium

Tel: $+32-3-280-3101$

Fax: +32-3-239 8658

E-mail: verhoeven.sarah@gmail.com 